\title{
QoS Analysis of Wireless Sensor Networks for Temperature and Humidity Monitoring and Control of Soybean Seed Storage Based IOT Using NodeMCU
}

\author{
Sindung HW Sasono ${ }^{1}$, Sri Kusumastuti ${ }^{1}$, Eko Supriyanto ${ }^{1}$, Sarono Widodo ${ }^{1}$, Dinda \\ Azizcha ${ }^{1}$ \\ ${ }^{1}$ Electrical Engineering Dept, Semarang State Polytechnic \\ ssindung@gmail.com
}

\begin{abstract}
Based on data from Central Bureau of Statistics in 2016 - 2020 soybean production deficit, the main factor is the decline in the quality of soybean seeds temperature and humidity sensitive. And so we need a system of monitoring and control of temperature and humidity container store soya beans. Wireless sensor network wireless technology that consists of a collection of sensor nodes distributed on a given area can support the communication between the sensor nodes using the system and sensor NodeMCU DHT11. This research was to analyze the WSN QoS monitoring system and temperature and humidity control soybean seed store container-based IOT using NodeMCU clients and coordinators are connected to an access point for sending data to the server in realtime. Tests carried out by putting the sensor node at 3 points with a distance variation of the coordinator and a data packet transmission interval. The test results obtained using a star topology indoor NLOS conditions optimal distance sensor node 3 which is 4 meters and the delivery interval $40 \mathrm{~s}$ with packet loss value of $0-20 \%$, delay $1.154-5,92 \mathrm{~s}$, jitter 0.241 to $7.57 \mathrm{~ms}$, and throughput 66.32 bits / s. WSN protocol IOT uses MQTT NodeMCU and goes well with a low throughput value is 529.81 bps to 544.85 bps can still generate a delay kualiatas 200.33 to $270.83 \mathrm{~ms}$ and packet loss from 0 to $1.284 \%$ which is good. 92s, jitter 0.241 to $7.57 \mathrm{~ms}$, and the throughput of 66.32 bits / s. WSN protocol IOT uses MQTT NodeMCU and goes well with a low throughput value is 529.81 bps to 544.85 bps can still generate a delay kualiatas 200.33 to $270.83 \mathrm{~ms}$ and packet loss from 0 to $1.284 \%$ which is good. $92 \mathrm{~s}$, jitter 0.241 to $7.57 \mathrm{~ms}$, and the throughput of 66.32 bits / s. WSN protocol IOT uses MQTT NodeMCU and goes well with a low throughput value is 529.81 bps to 544.85 bps can still generate a delay kualiatas 200.33 to $270.83 \mathrm{~ms}$ and packet loss from 0 to $1.284 \%$ which is good.
\end{abstract}

Index Terms — WSN, NodeMCU ESP8266, packet loss, delay, throughput, jitter, star

when compared with those in the highlands. This is due to the different environmental conditions that soybean production in

\section{PRELIMINARY}

Based on data from Central Bureau of Statistics in 2015, the food consumption of soybean like tempeh in Indonesia amounted to $6.99 \mathrm{~kg}$ and $7.51 \mathrm{~kg}$ per year out. But the number of commodities imported soybean in Indonesia is still high at $67.28 \%$ or 1.96 tons. Based on projected results, expected in the years 2016 - 2020 the balance of production and consumption of soy deficit average - an average of $36.95 \%$ per year. The main factor of high soybean import figures are Indonesia as soybean cultivation state deteriorated quality of soybean seed production [1].

Good soybean harvest period towards the next planting season soybean seed required waiting period of approximately 6 months. During the waiting time, it takes the place of storage to support the soybean seed to maintain its quality during those 6 months. Soy is used for seed storage that is not directly used.

In Indonesia, the location of soy farms in the highlands and lowlands. In the lowland produce soybean seeds are less well lowland areas more quickly degenerated seed during storage so mengurrangi availability of high quality seeds. Yellow soybean seed storage at low temperature (200C - 230C) are able to maintain high quality of the seed is stored for six months [2].

To overcome these problems, it can be built monitoring system and temperature control soybean seed store containers with technology

IOT (Internet of Things). The problems come up again if in an integral storage system within the necessary existence of many sensor nodes. To connect all those sensors that can communicate a need for a technology WSN (Wireless Sensor Network). WSN technology is a merger between sensing technology (sensing) and network technology (networks). With the implementation of the IOT WSN can overcome the problems of communication between the nodes and the needs of the user separated by distance. This system can work without checking the temperature directly in other words can be done remotely and real-time with minimum cost or expense.

\section{RELATED RESEARCH}


System monitoring and control soybean seed storage temperature using a system of Wireless Sensor Network (WSN) are connected through the Internet to facilitate the work of observing the state of the container store indoors soybean seeds over long distances.

There are few studies regarding the monitoring of an environment by using sensors connected to the Internet-based system of IOT. In a study [3], entitled "Analysis of System Performance Monitoring Temperature And Humidity Based Wireless Sensor Network" is said that to support the agricultural resources needed one technology that is monitoring system temperature and humidity environment based wireless sensor network with good performance that is comparable to paramer observed. From the test results he had done showed that all systems can work well with a $100 \%$ success percentage DHT11.

In a study [4], entitled "Implementation and Analysis of Wireless Sensor Network for Monitoring Temperature, humidity and $\mathrm{CO} 2$ levels on the room" to discuss the quality of the network to multiple nodes and different types of topologies. Results from the design and realization of a sensor system that is the result of the calculation of the value of the temperature, humidity and $\mathrm{CO} 2$ levels wirelessly with a distance of 61.84 meters optimally obtained without a router nodes and more than 65 meters with a router node in indoor. In this study, carried out by using the XBee as the transmission medium.

In the study [5], entitled "Design of Wireless Sensor Network Based on ZigBee protocol And Gsm To Air Pollution Monitoring System"

To measure and communicate information in real-time can use wireless technology sensor network (WSN). This research proposes the development of an air pollution monitoring system distributed wirelessly using the ZigBee protocol and GSM networks.

In all three of these studies have not discussed about how the influence of WSN (Wireless Sensor Network) affecting the data transmission of sensor data delivery to the server by using one of which ESP 8266 IOT platform v1.0. So in this final testing how WSN (Wireless Sensor Network) good to be implemented in the system of monitoring and control soybean seed storage.

Temperature and humidity are optimal for storage of soybean seeds is a temperature of $20^{\circ} \mathrm{C}-220^{\circ} \mathrm{C}$ and a humidity of $64 \%-80 \%$.

\section{DESCRIPTION SYSTEM}

Making the wrieless sensor network monitoring and control system using the IOT-based NodeMCU aims to monitor and control soybean seed storage temperature. In this study conducted at ambient conditions with NLOS (Non Line of Sight) with a barrier in the form of brick walls, gypsum, and glass doors that can affect the quality of WSN generated. An overview of the overall system shown in Figure 1.

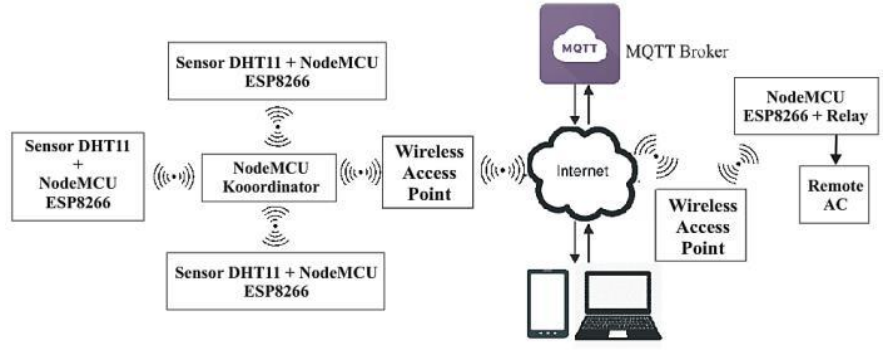

Figure 1. Overall Monitoring and Control System

In the container monitoring system saved soybean seeds with IOT technology using a temperature sensor that is placed on three points of the room. The temperature sensor is connected with the brain ESP8266 NodeMCU monitoring program. Furthermore, the temperature sensor is connected to this ESP8266 NodeMCU called a sensor node. In the storage space control system using only soy beans ESP8266 NodeMCU connected to the Internet (access point) in order to take control of the room temperature in any place through MQTT Broker.

The control system can be taken after seeing the results of monitoring of temperature data. The control system was made with the AC power control on the storage space using a laptop or a smartphone that has been connected to the Internet. When the user presses on the control buttons on the smartphone display MQTT Broker, the system receives the commands and perform the command to turn on the $\mathrm{AC}$ in the storage space.

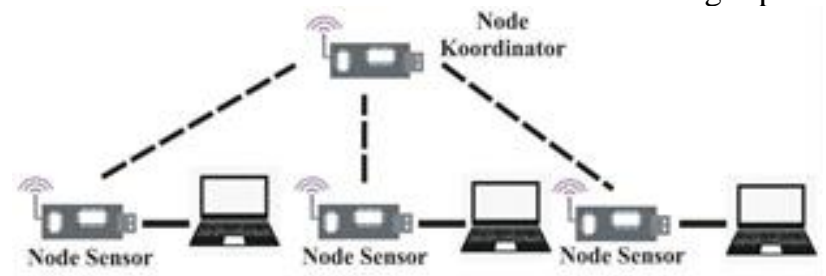

Figure 2. Star Topology WSN In System

Testing is done by programming the data transmission from the device NodeMCU ESP8266 as sensor nodes toward NodeMCU device ESP8266 as the coordinator node and take the delivery of data capture using software TeraTerm. Of data capture that has been drawn, can be calculated the value of QoS delay, throughput, jitter, and packet loss occurs. Testing using wireshark software is used to see if the connection between the sensor nodes are connected to the sever NodeMCU above MQTT protocol in real-time. 
In a star topology testing is done by connecting three sensor nodes simultaneously to the coordinator node connected to the server via the internet broker Mqtt provided by the wireless access point. Testing with a star topology aims to test the performance of NodeMCU to serve three nodes simultaneously at once to get the quality of the resulting network system using ESP8266 NodeMCU in NLOS conditions.

Node sensor formed are several units that mikokontroler unit, transceiver unit, battery pack, and a sensor unit. This study uses MCU Node ESP8266 as microcontrollers and also the receiver on the sensor node. ESP8266 MCU node already has a wireless module Wi-Fi $802.11 \mathrm{~b} / \mathrm{g} / \mathrm{n}$, so it can directly transmit and receive data wirelessly. In this paper, the temperature and humidity penyimpaan taken by the temperature and humidity sensors DHT11.

Node coordinator as recipient of the data from the sensor nodes by using MCU Node ESP8266.

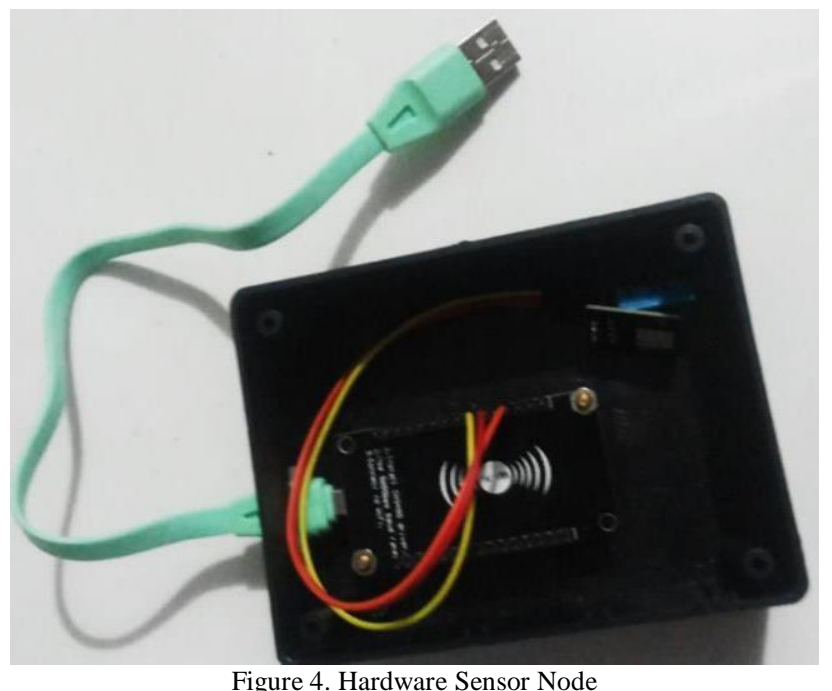

The control circuit uses NodeMCU ESP8266 as the brains of the control program. The technique used to control the temperature of the storage environment using the relay to replace the remote button function $\mathrm{AC}$ (Air Conditioner) associated with NodeMCU ESP8266 as a driver. MQTT control circuit connected to the broker with an internet connection, so that control can be done via smartphone or computer wherever place.

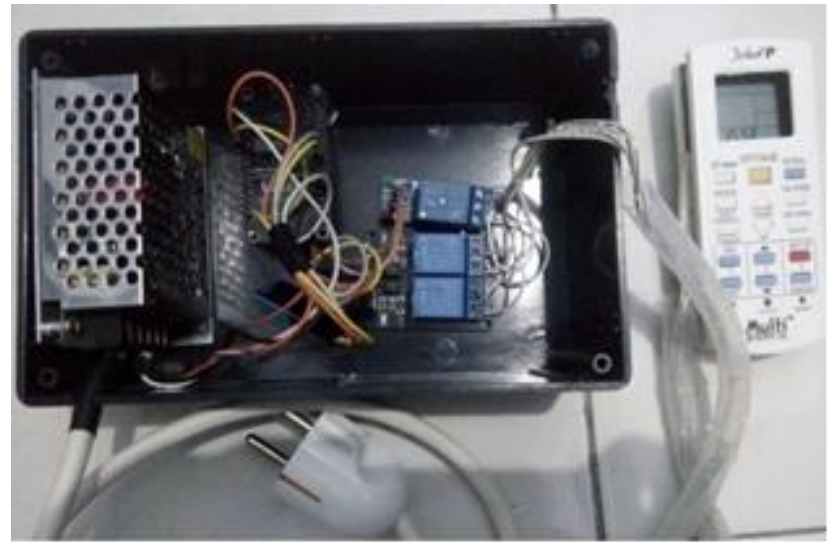

Figure 5. Hardware Node Control

\section{A. Software}

The sensor nodes, the coordinator, and a control node configured so that the program can be run using the Arduino IDE software to upload the program to NodeMCU ESP8266. Upload Esp8266 program to Node MCU with Arduino IDE already installed library of previous node ESP8266 MCU. The configuration for $\mathrm{Wi}-\mathrm{Fi}$ and wireless connections, as well as the program via the Arduino IDE. And then configure the program to connect with MQTT.Diagram program flow monitoring system can be seen in Figure 6 and Figure 7 for the control system. In this system uses three sensors to obtain a sample of the actual data of soybean seed storage. MCU node will send sensor data generated toward MQTT coordinator then forwarded to the broker to the Internet network from the access point,

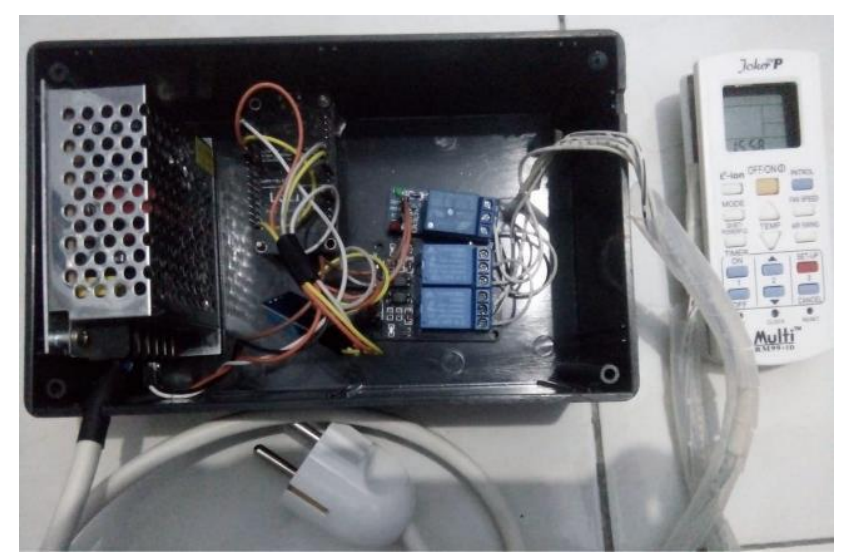




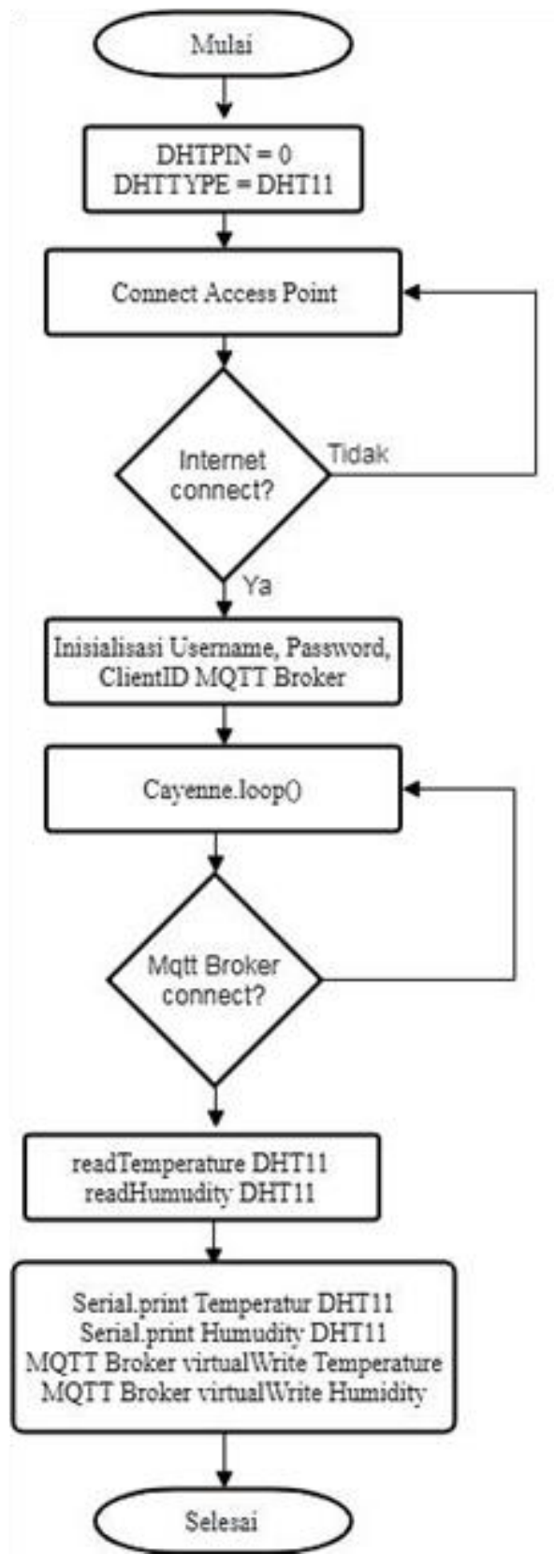

(a)

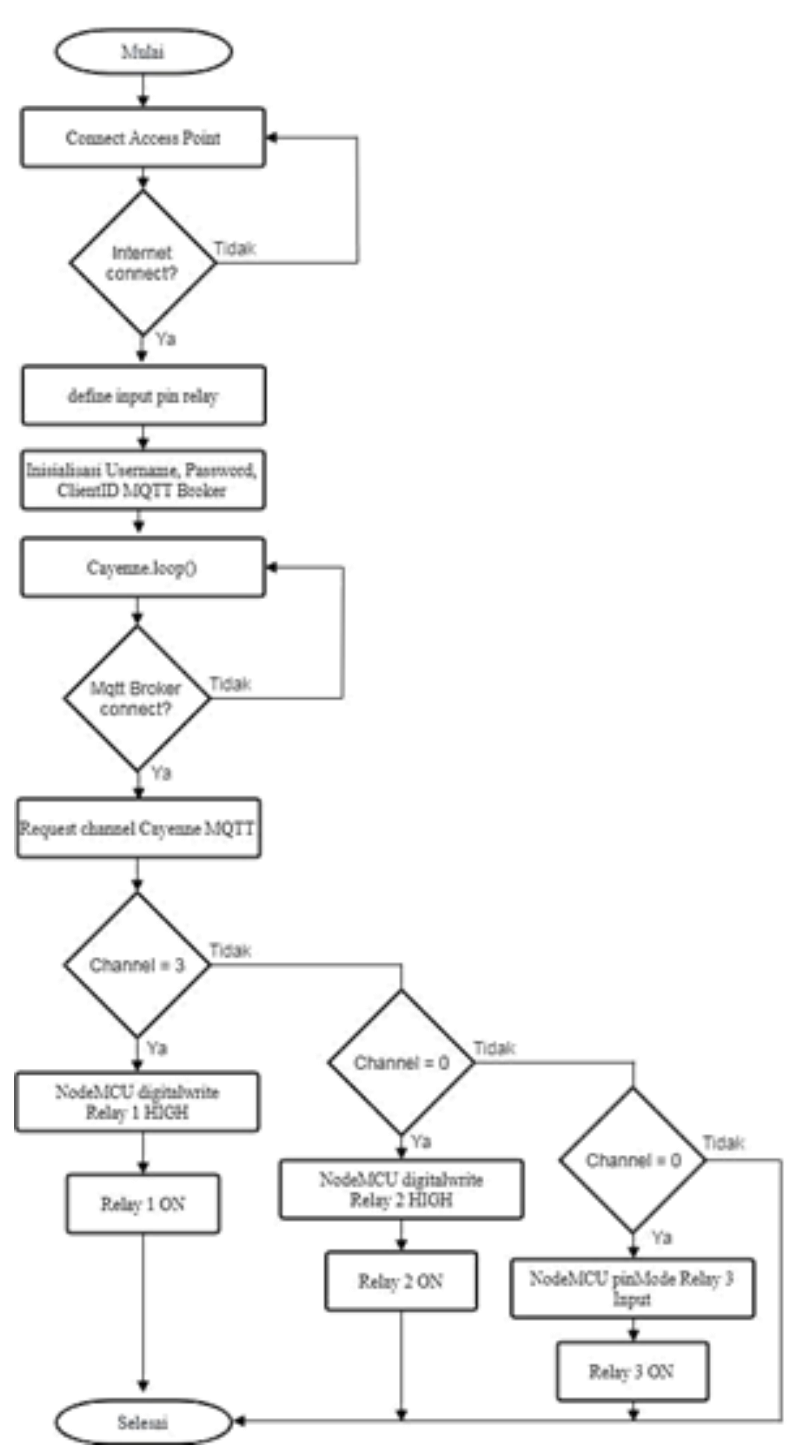

(b)

Figure 6 (a) Flowchart of Monitoring System (b) Control System

B. QoS (Quality of Service)

Here's a network quality parameters as indicators of the sensor nodes is said to be good or bad in producing quality network with a certain distance in the application system of monitoring and control soybean seed storage.

1) Packet Loss

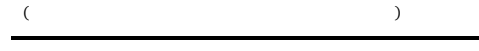

packet Loss is a parameter that describes a condition that indicates the total number of packets lost, can occur due to collision and congestion on the network and it affects all applications for retransmission will reduce overall network 
efficiency despite ample amount of bandwidth available for such applications.

\section{2) Delay / Latency}

Delay / latencydefined as the length of time required by the data packet to a destination. Latency is the time it takes the data to travel from origin to destination. Latency can be affected by distance, physical media or also a lengthy process.

\section{3) jitter}

jitter is a variation of the delay, where there is a delay difference on the package - a package that is sent to the flow of data packets (Data Flow) are the same. Jitter is usually called delay variation, closely related to latency, which indicates the number of delay variation on taransmisi data on the network. Delay queues on routers can cause jitter.

\section{4) throughput}

throughputa number of successful packets sent at regular intervals. Measurements on throughput is to divide the speed (rate) effective data transfer at a certain time interval ( $\mathrm{s}$ ) and is measured in bps (bits per second) or Mbps (mega bits per second).

\section{RESULTS AND ANALYSIS}

Testing in this thesis aims to determine network performance is generated by using NodeMCU to indoor conditions and NLOS (Non Line of Sight), which can be evaluated from the QoS parameters. QoS parameters are observed delay, throughput, packet loss, and jitter.

In a star topology variables pengkoneksian testing the distance digunkaan coordinator node to node. Tests carried out in the room with the barriers of the bulkhead. Data is collected on indoor conditions in Eastern Telecommunications Lab building Polytechnic State Semarang .. Condition of the building has several rooms with insulation barrier between the room in the form of gysum and cement.

Stage data processing done is:

The data processing method used is completely randomized factorial design to determine the effect of changes due to two factors: the distance sensor nodes and sensor nodes send time interval of the QoS value of WSN in system testing.
1. Before obtaining the results of calculation, to determine in advance the formulation of hypotheses that could happen is as follows:

a. The influence of the distance factor sensor nodes

$\mathrm{HO}$ : There is no difference in the value of QoS due to the effect of distance sensor nodes.

Ha : There is a difference in the value of QoS due to the effect of distance sensor nodes.

b. Influence factors interval to send the data

$\mathrm{HO}$ : There is no difference in the value of QoS due to delivery interval data.

Ha : There are different grades of QoS due to delivery interval data.

c. The influence of the distance factor and the interval sensor nodes send the data

H0: Factors distance sensor nodes do not interact with data transmission intervals.

Ha: Factors distance sensor nodes interact with the data transmission interval.

1. Determining the significance level of $5 \%(\alpha=0.05)$

2. Criteria for rejection $\mathrm{H}_{0} \mathrm{H} 0$ is rejected if sig $<0.05$

I. Heading Sensor Node Connection Testing Coordinator

Tests carried out by programming the test connection with the sensor node ping program, this test aims to determine the furthest distance sensor node and coordinator node can communicate using ESP8266 NodeMCU in indoor and NLOS conditions.

Table 1 Results of Tests Sensor Node Connection with Node Coordinator (NodeMCU ESP8266)

\begin{tabular}{ccc}
\hline No. & testing Distance & Description success \\
\hline 1 & $2 \mathrm{~m}$ & succeed \\
2 & $4 \mathrm{~m}$ & succeed \\
3 & $6 \mathrm{~m}$ & succeed \\
4 & $8 \mathrm{~m}$ & succeed \\
5 & $10 \mathrm{~m}$ & succeed \\
6 & $12 \mathrm{~m}$ & succeed \\
7 & $14 \mathrm{~m}$ & Not successful
\end{tabular}



8
$16 \mathrm{~m}$
Not successful

Sensor node sends a ping command to the coordinator node to determine whether successful or individual sensor nodes can be connected to the coordinator node to the next can transmit sensor data. When the coordinator node to respond in the form of reply the sensor node and coordinator node can still be connected. But if the Coordinator node response request timed out.

\section{Star Topology WSN QoS testing}

QoS Testing performed using software TeraTerm. NodeMCU ESP8266 as sensor nodes connected to the laptop using the usb cable. The sensor nodes are then connected to an access point wirelessly on the NodeMCU ESP8266 as WSN star topology of the system of monitoring and control soybean seed storage. Used variance and interval distance data transmission as a sensor node testing parameters.

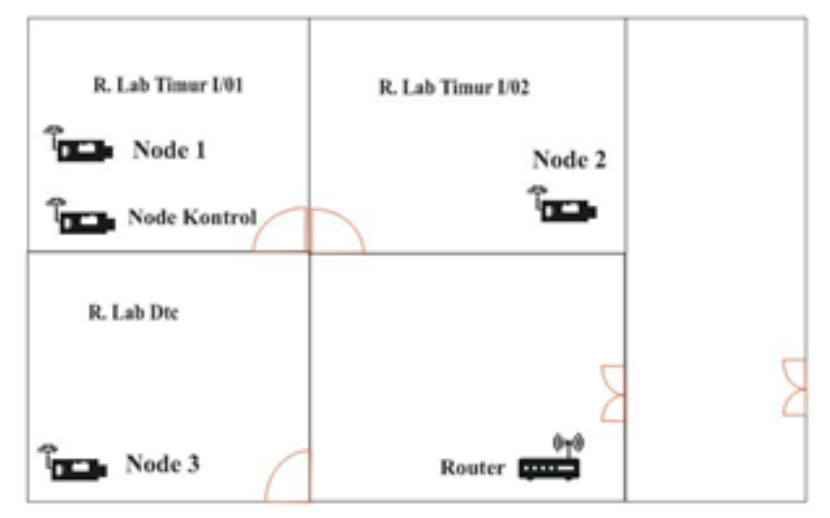

Figure 7 Placement Node Topology Star

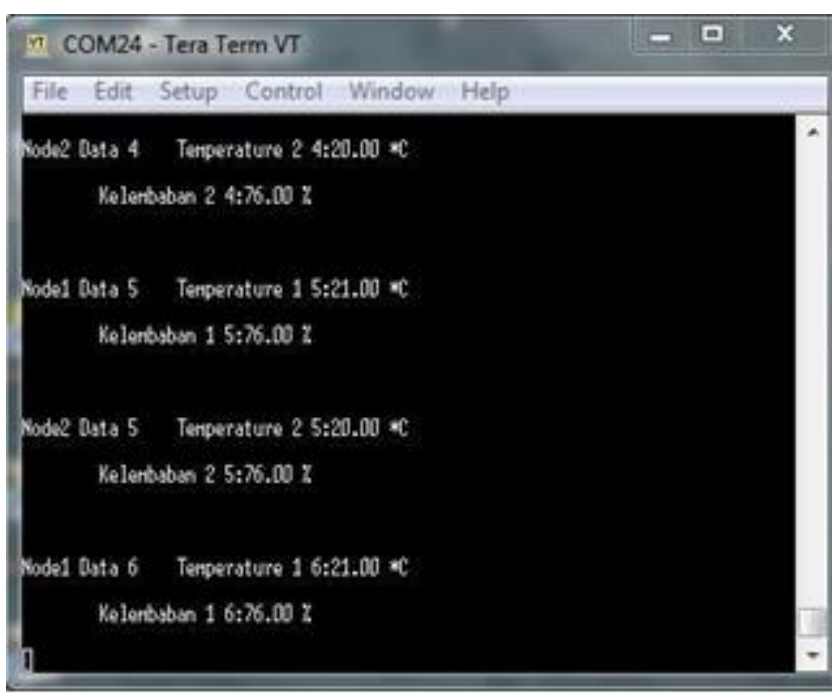

Figure 8 Capture Data Delivery Program

QoS is used for testing pemgiriman program of data by sensor nodes to the coordinator node on each test in the capture using software TeraTerm shown in Figure 8.

Table 2 Results of Testing Topology Star (NodeMCU ESP8266)

\begin{tabular}{ccccccc}
\hline & trial to & \multicolumn{5}{c}{ QoS value } \\
\hline Dista & Inter- & Packet & Dela & Jitter & put \\
nce & val & node & loss & y (s) & (s) & (bps) \\
(m) & send & & $(\%)$ & & & \\
& (s) & & & & & \\
\hline
\end{tabular}

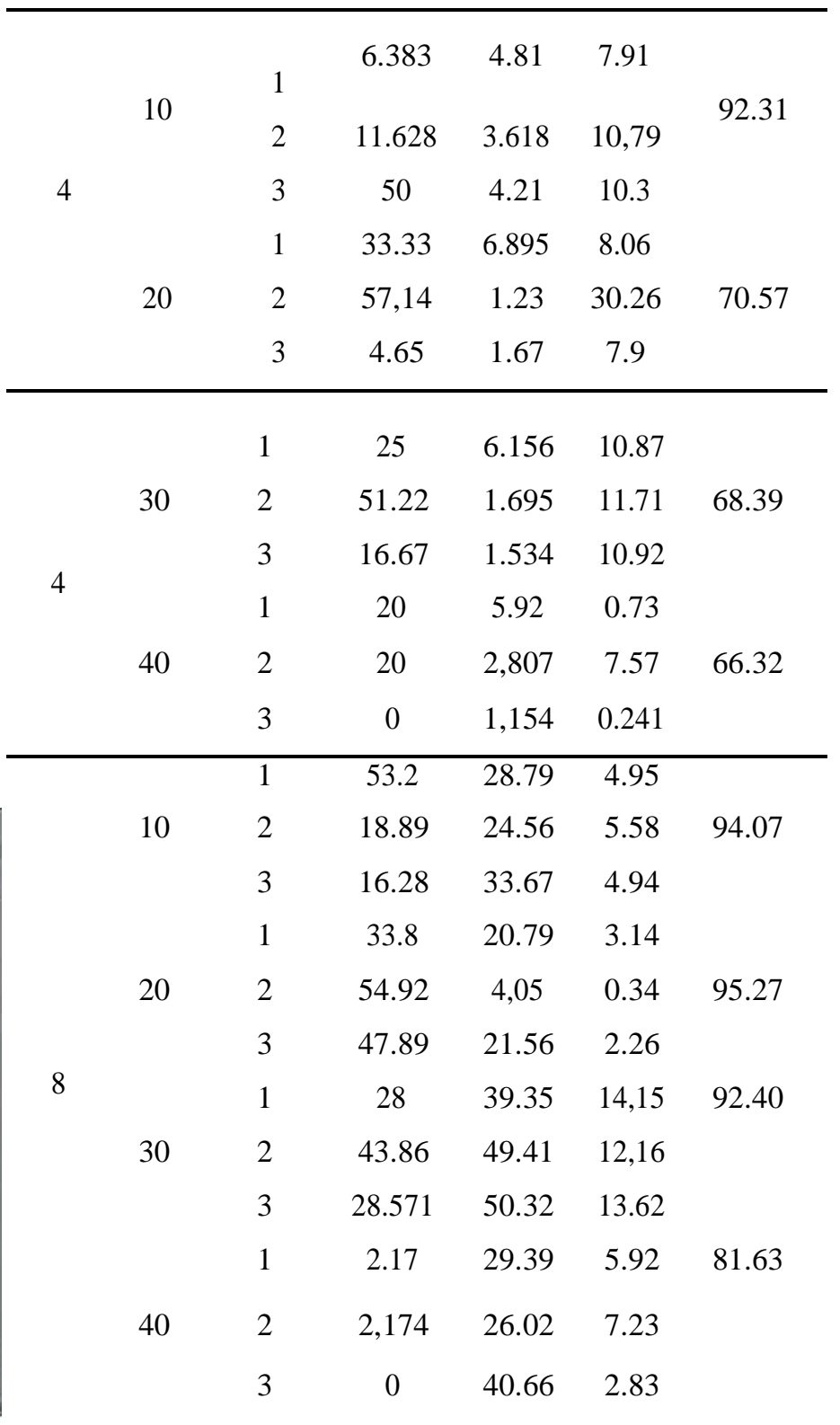

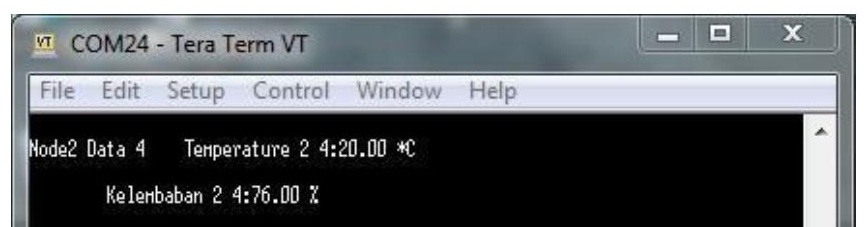




\begin{tabular}{|c|c|c|c|c|c|c|}
\hline \multirow{12}{*}{12} & \multirow{3}{*}{10} & 1 & 50 & 40.74 & 8.107 & \multirow{3}{*}{97.09} \\
\hline & & 2 & 57.143 & 27.16 & 8.55 & \\
\hline & & 3 & 54.762 & 40.36 & 6,63 & \\
\hline & \multirow{4}{*}{20} & 1 & 10.714 & 108.3 & 32.94 & \multirow{4}{*}{91.20} \\
\hline & & 2 & 26.786 & 88.10 & 30.12 & \\
\hline & & 3 & 68.056 & 25.52 & 8.517 & \\
\hline & & 1 & 60.94 & 26.55 & 4.833 & \\
\hline & \multirow[t]{3}{*}{30} & 2 & 59.375 & 8.78 & 1,486 & \multirow[t]{2}{*}{78.62} \\
\hline & & 3 & 31.25 & 18.04 & 1.02 & \\
\hline & & 1 & 78.33 & 60.75 & 274.1 & \multirow{3}{*}{37.90} \\
\hline & \multirow[t]{2}{*}{40} & 2 & 46.67 & 5,034 & 2,48 & \\
\hline & & 3 & 84.21 & 9.23 & 0.198 & \\
\hline
\end{tabular}

1) Packet Loss

Based on the calculation completely randomized factorial design can be seen the influence of the data transmission interval and distance of each - each sensor node to the coordinator node of the value of packet loss.

Two-Way ANOVA: Loss versus Interval; Node

$\begin{array}{lrrrrr}\text { Source } & \text { DE } & \text { SS } & \text { MS } & \text { F } & \text { P } \\ \text { Interva1 } & 3 & 574,1 & 191,38 & 0,59 & 0,630 \\ \text { Node } & 2 & 5572,1 & 2786,05 & 8,54 & 0,002 \\ \text { Interaction } & 6 & 5030,2 & 838,36 & 2,57 & 0,046 \\ \text { Error } & 24 & 7828,0 & 326,16 & & \\ \text { Iotal } & 35 & 19004,4 & & & \end{array}$

Figure 9 Calculation of Effect of Interval and Distance Against Packet Loss Using Minitab

In Figure 9 it can be seen that the value of packet loss indoor NLOS conditions generated within the sensor node to significant influence by the indicated value of $\mathrm{P}<0.05$ so that $\mathrm{HO}$ is rejected causing no difference packet loss results due to the effect of distance sensor nodes. As for the data transmission interval did not significantly affect the value of packet loss generated by the indicated value of $\mathrm{P}>0.05$ so $\mathrm{H} 0$ is no significant difference in the effect of packet loss due to data transmission intervals. Factors distance sensor nodes interact with the data transmission interval factor indicated by the value of $\mathrm{P}<0.05$.

In the figure 10 is a graph of the output sensor node distance factor influence on the value of packet loss on Minitab, note that the value of packet loss increased with the increase within each - each sensor node to the coordinator node.

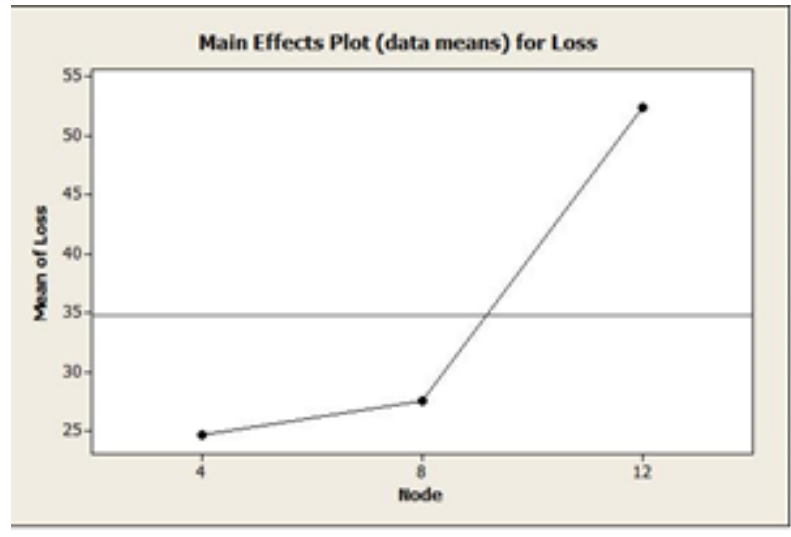

Figure 10 Influence Factors Against Distance Sensor Node Packet Loss

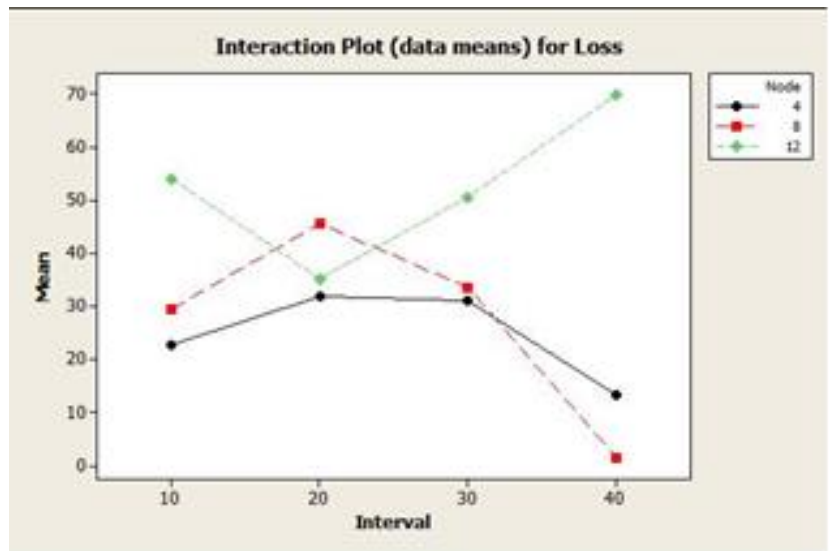

Figure 11 Figure Interaction To Interval Time Shipping and Proximity Sensor Node Against Packet Loss

Characteristics of the interaction between the sensor nodes at intervals distance data transmission against packet loss can be seen in Figure 11. Based on the graph plots Minitab interaction on the highest value of packet loss occurs during data transmission is given 40 second intervals with a 12 meter distance sensor nodes. As for the value of the lowest packet loss occurs during the given interval of 40 seconds of sensor nodes with a distance of 8 meters.

2) Delay

Based on the calculation completely randomized factorial design can be seen the influence of the data transmission interval and distance of each - each sensor node to the coordinator node to the delay value.

\section{Two-way ANOVA: Delay versus Interval; Node}

$\begin{array}{lrrrrr}\text { Source } & \text { DF } & \text { SS } & \text { MS } & \text { F } & \text { P } \\ \text { Interval } & 3 & 595,0 & 198,35 & 0,74 & 0,537 \\ \text { Node } & 2 & 8021,4 & 4010,69 & 15,02 & 0,000 \\ \text { Interaction } & 6 & 6483,0 & 1080,51 & 4,05 & 0,006 \\ \text { Error } & 24 & 6408,1 & 267,00 & & \\ \text { Total } & 35 & 21507,6 & & & \end{array}$

Figure 12 Calculation of Effect of Interval and Distance Against Delay Using Minitab 
In figure 12 it can be seen that the delay value of indoor NLOS conditions generated within the sensor node to significant influence by the indicated value of $\mathrm{P}<0.05$ so that $\mathrm{HO}$ is rejected causing no difference delay results due to the effect of distance sensor nodes. As for the data transmission interval did not significantly affect the value of delay generated by the indicated value of $\mathrm{P}>0.05$ so $\mathrm{H} 0$ is no significant difference of delay due to the influence of the data transmission interval. Factors distance sensor nodes interact with the data transmission interval factor indicated with a $\mathrm{P}$ value $<0.05$ so that the interaction that occurs is the sensor nodes increase the distance factor affects the interval factor.

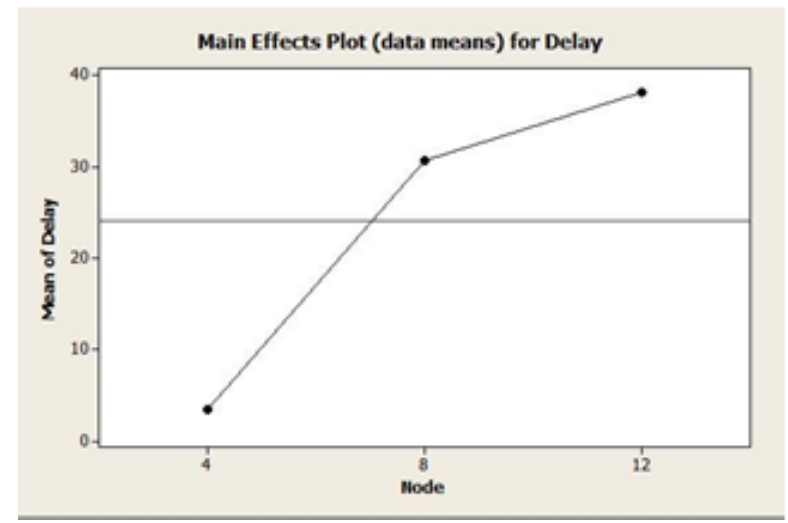

Figure 13 Influence Factors Against Delay Distance Sensor Node

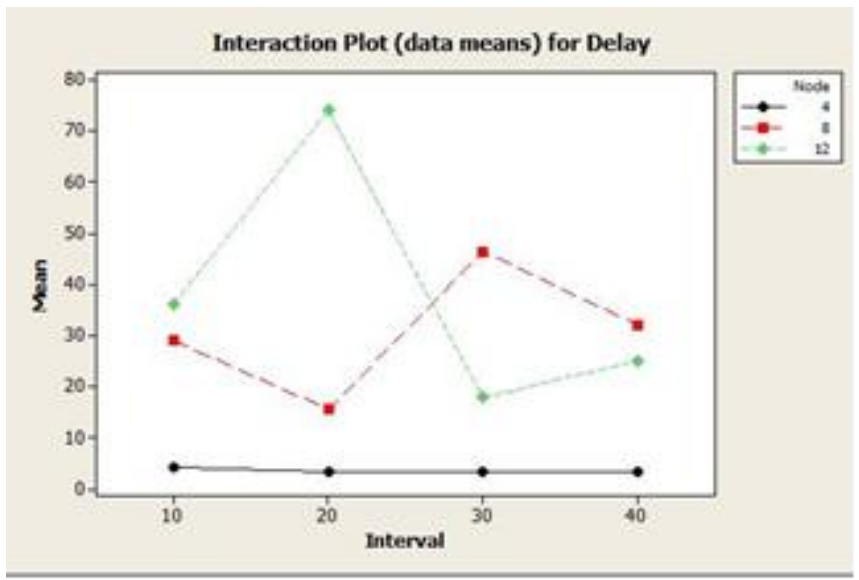

Figure 14 Graph Interaction To Interval Time Shipping and Proximity Sensor Node Against Delay

In figure 13 is a graph of the output sensor node distance factor influence on the value of delay in Minitab, it is known that the delay value increased with the increase within each each sensor node to the coordinator node.

Characteristics of the interaction between the sensor nodes at intervals distance data transmission against the delay can be seen in Figure 14. Based on the interaction Minitab graphs plot the highest delay value occurs during data transmission is given 20 second interval with 12 meters distance sensor nodes. As for the value of the lowest delay occurs when given 40 second intervals with the sensor node distance of 4 meters.

\section{3) Jitter}

Based on the calculation completely randomized factorial design can be seen the influence of the data transmission interval and distance of each - each sensor node to the coordinator node to the value of jitter.

\section{Two-way ANOVA: Jitter versus Interval; Node}

$\begin{array}{lrrrrr}\text { Source } & \text { DE } & \text { SS } & \text { MS } & \text { F } & \text { P } \\ \text { Interval } & 3 & 3882,8 & 1294,27 & 0,62 & 0,611 \\ \text { Node } & 2 & 4476,1 & 2238,03 & 1,07 & 0,360 \\ \text { Interaction } & 6 & 12037,2 & 2006,19 & 0,96 & 0,475 \\ \text { Error } & 24 & 50326,7 & 2096,95 & & \\ \text { Total } & 35 & 70722,7 & & & \end{array}$

Figure 15 Calculation of Effect of Interval and Distance To Jitter Using Minitab

In the figure 15 can be seen that the jitter value of indoor NLOS conditions generated for the distance sensor nodes indicated no significant effect with a $\mathrm{P}$ value $>0.05$ so that $\mathrm{H} 0$ is accepted that there is no difference jitter due to the effect of distance sensor nodes. For data transmission interval did not significantly affect the value of jitter generated with the indicated value of $\mathrm{P}>0.05$ so $\mathrm{H} 0$ is no significant difference jitter due to the influence of the data transmission interval. Factors distance sensor nodes do not interact with data transmission interval factor indicated with a $\mathrm{P}$ value $>0.05$.

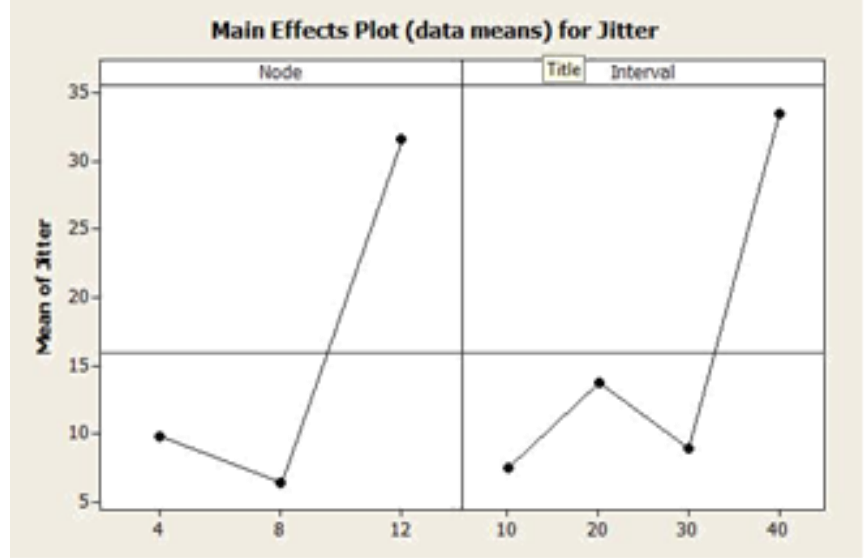

Figure 16 Influence Factors and Interval Distance Sensor Node Delivery Against Jitter

In the figure 16 is an output chart the influence of sensor nodes and interval distance data transmission against jitter value in Minitab, it is known that the jitter value is not stable for each increment distance sensor nodes or delivery interval. Due to the jitter value is affected by the variance of delay, so that will greatly affect the value of delay jitter is unstable.

\section{4) Throughput}


Based on the calculation completely randomized factorial design can be seen the influence of the data transmission interval and distance of each - each sensor node to the coordinator node to the throughput value.

\section{Two-way ANOVA: Throughput versus Interval; Node}

$\begin{array}{lrrrrr}\text { Source } & \text { DE } & \text { SS } & \text { MS } & F & P \\ \text { Interval } & 3 & 1701,42 & 567,139 & 3,45 & 0,092 \\ \text { Node } & 2 & 650,70 & 325,350 & 1,98 & 0,219 \\ \text { Error } & 6 & 986,88 & 164,480 & & \\ \text { Total } & 11 & 3339,00 & & & \end{array}$

Figure 17 Calculation of Effect of Interval and Distance Against Throughput Using Minitab

In the figure 17 it can be seen that the throughput of indoor NLOS conditions generated for the distance sensor nodes indicated no significant effect on the value of $\mathrm{P}>0.05$. As for the data transmission interval did not significantly affect the throughput generated by the indicated value of $\mathrm{P}>0.05$, but is still close to its value significantly.

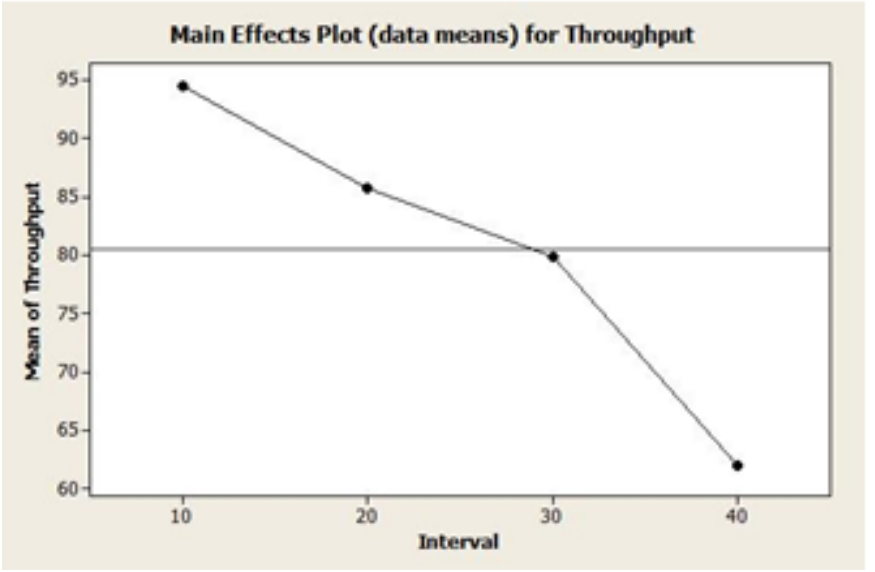

Figure 18. Effect of Interval Inquiry Against Data Throughput

In the figure 18 is a graph of the output intervals of factors influence the delivery influences the throughput value in Minitab, it is known that the throughput decreases with the increase of data transmission intervals, due to the increasingly distant intervals, the data received by the less by the coordinator node.

\section{Testing Coordinator Connection Broker Towards MQTT}

The overall system in this thesis using a star topology where there are three sensor nodes connected to the coordinator node which further data is transmitted to the server (mqtt broker) by using an Internet connection from the access point. WSN QoS value in the system is measured using wireshark software, where each sensor node sends the data reading of each - each headed mqtt broker.

Testing delay for the whole system is done by calculating the time publish until ack appear. Delay value can be seen in the file properties and the results graph delay of each node. Delay value have been affected by the quality of the Internet network service that is being used during the test. At the time of the test used one type of internet service providers only. Value - average delay produced is proportional to the value of packet loss that occurred on each - each sensor node.

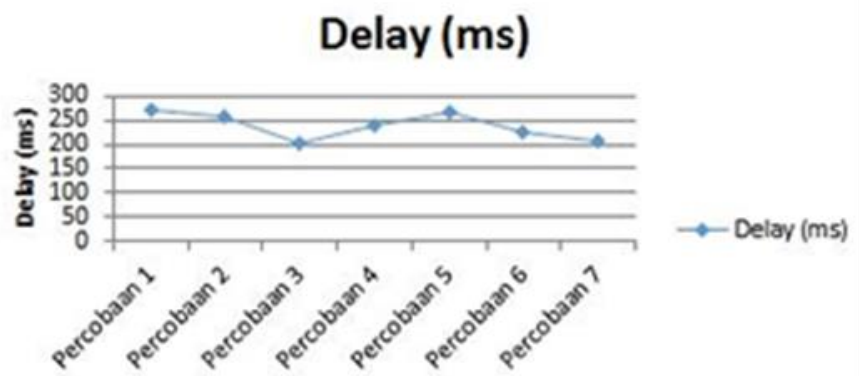

Figure 19 Graph Delay Overall Test Result System

Value delay in trial 5 is the highest compared to other experiments that $268.2848665 \mathrm{~ms}$, because at the time of the experiment 5 has the highest delay value when compared to other experiments.

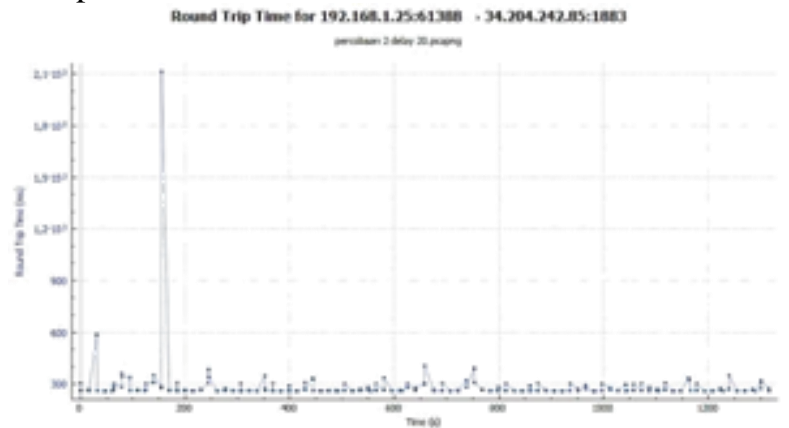

Figure 20 Graph Capture Wireshark test of Results Delay

Overall, the value of the system delay is the delay category is quite good. Figure 20 shows the graph displayed on the software delay wireshark. The graph shows the spike indicates the missing packet or retransmission packet which is when they publish the data has reached approximately 2 second delay time, so the system asks for retransmission of the packet is assumed that the package was lost. Packet loss of the entire system can see the results of wireshark capture samples that have been taken. The resulting packet loss can be determined by counting on wireshark packet retransmission requests are also done in [6].

Can be seen in the graph 21 that the value of packet loss irregular ups and downs, but overall the results of testing and calculation of the value of packet loss is still in the very good category for each sensor node. Packet Loss is also affected by the delay value at the time. 


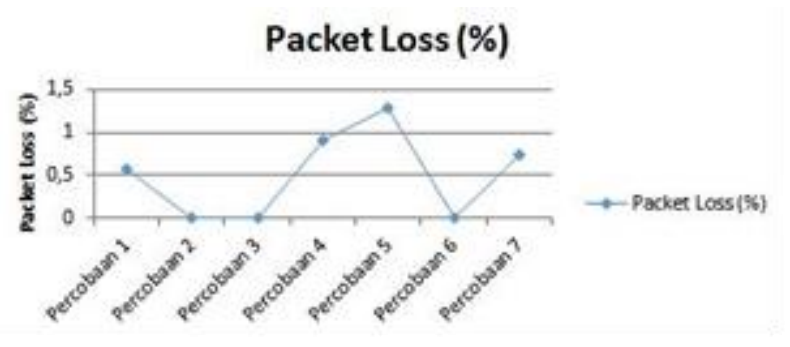

Figure 21 Graph Packet Loss Test Result Towards Broker

\section{Throughput (bits/s)}

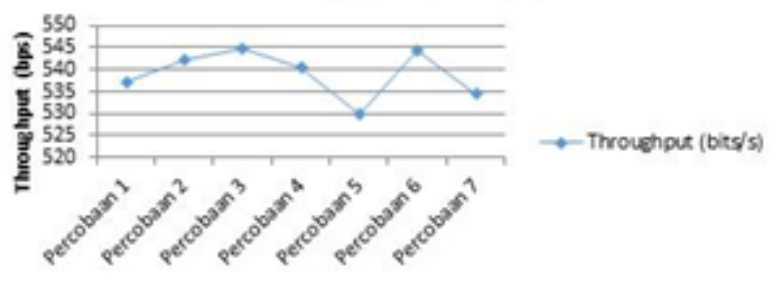

Figure 22 Graph Results Overall Throughput Testing System

Throughput value is inversely proportional to the value of delay and packet loss generated in each test. The throughput value is $544.85 \mathrm{bps}$ is on trial 3 where the absence of packet loss at the moment, while the smallest value is 529.8132 bps throughput that is on trial 5 where the highest value of packet loss at the time of trial. With these values are relatively low, mqtt protocol can still transmit data packets with packet loss quality is still good.

\section{Testing with a Control Node Connection Broker}

Testing is done by pressing the control button comprising each of the on / off button Rev temperature, and the temperature down button. Each of these buttons can generate QoS values read by Wireshark.

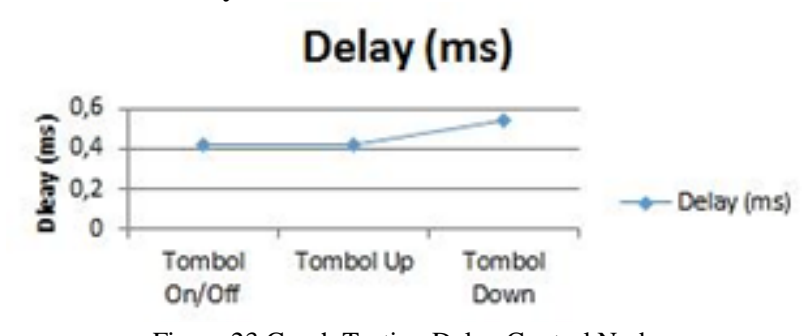

Figure 23 Graph Testing Delay Control Node

Almost the same delay value for each key if it does not happen gangguang internet services provided by wireless access point.

\begin{tabular}{|c|c|c|c|}
\hline Measirements & Caobred & Desolyed & Myked \\
\hline Padkets & 4997 & $258(5.2 \%)$ & - \\
\hline Tme span, s & 435.512 & 425,993 & - \\
\hline Average pps & 11.5 & 0.6 & - \\
\hline Average podket size, 8 & 269 & 142 & - \\
\hline Bytes & 1345430 & $36762(2.7 \%)$ & 0 \\
\hline Average bytes/s & 3089 & 86 & - \\
\hline Average bits/s & $24 k$ & 690 & - \\
\hline
\end{tabular}

Figure 24 Capture Statistics On Wireshark
In testing the control node, throughput is not far from the throughput obtained when the data mengikimkan coordinator node towards mqtt broker that is equal to $690 \mathrm{bps}$.

\section{CONCLUSION}

From the experimental results and the manufacture of systems in this study can be taken some conclusions as follows:

1. Mqtt protocol can run well on a system of monitoring and control soybean seed storage can work in realtime and can be accessed from anywhere via an Internet connection.

2. The most optimal maximum distance indoor NLOS conditions using an access point NodeMCU ESP8266 is 12 meters.

3. QoS Quality of packet loss and delay of a star topology network is affected by the distance sensor node to the coordinator node. QoS Quality of jitter is not affected by the distance factor and the sensor nodes to the coordinator the data transmission interval factor. On star topology resulting throughput value declines with data transmission intervals.

4. Interval delivery good enough to support communication NodeMCU sensor node by node coordinator using the star topology in indoor conditions NLOS is 40 second to prevent accumulation of data and the placement of the sensor node at a distance of 4 meters with a value of packet 
[3]. Royan, D., Primananda, R., \& Kurniawan, W. (2017). Analisis Performa Sistem Pemantauan Suhu Dan Kelembaban Berbasis Wireless Sensor Network. Jurnal Pengembangan Teknologi loss $0-20 \%$, delay 1.154 to 5,92 s, jitter 0.241 to 7.57

$\mathrm{ms}$, and the throughput of $66.32 \mathrm{bits} / \mathrm{s}$. Informasi Dan Ilmu Komputer Vol. 1, No. 12, Desember 2017, HIm. 1865-1874 e-ISSN:, 1(12), 1865-1874.

[4]. Kurniawan, A., Munadi, R., \& Mayasari, R. (2016). Implementasi dan Analisa Jaringan Wireless Sensor Untuk Monitoring Suhu , Kelembaban dan Kadar CO2 Pada Ruangan. Seminar Nasional

\section{References}

[1]. Suwandi. (2016). Outlook Komoditas Pertanian Tanaman Pangan Kedelai, 85.

[2]. Purwanti, Setyastuti, I. P. (2004). Kajian Suhu Ruang Simpan Terhadap Kualitas Benih Kedelai Hitam Dan Kedelai Kuning. Ilmu Pertanian, 11(1), 22-31.

[5]. Fuad, M. (2015). Rancang Bangun Wireless Sensor Network Berbasis Protokol Zigbee Dan GSM Untuk Sistem Pemantauan Polusi Udara. Institut Pertanian Bogor.

[6]. Lee, S., Kim, H., Hong, D. K., \& Ju, H. (2013). Correlation Analysis of MQTT Loss and Delay According to QoS Level. International Conference on Information Networking 